\title{
Violencia intrafamiliar según características sociodemográficas en una población pediátrica
}

\begin{abstract}
Cristina Haydée Arrom Suhurt ${ }^{\mathrm{I}}$, Andrés Claudelino Arce Ramírez ${ }^{\mathrm{I}}$, Carmen Marina Arrom ${ }^{\mathrm{II}}$, María del Pilar Fresco Arrom ${ }^{\mathrm{I}}$, María Auxiliadora Arrom ${ }^{\mathrm{II}}$, Mirta Mercedes Romero Núñez ${ }^{\mathrm{I}}$

I Cátedra de Psiquiatria, Facultad de Ciencias Médicas de la Universidad Nacional de Asunción UNA - Paraguay II Centro para el Desarrollo de la Investigación Científica (CEDIC).
\end{abstract}

Cómo referenciar este artículo/ How to reference this article:
Arrom C, Arce A, Arrom CM, Fresco MP, Arrom MA, Romero M. Violencia Intrafamiliar según características sociodemográficas en población pediátrica. Mem. Inst. Investig. Cienc. Salud. $2015 ; 13(3): 58-63$

\section{RE S U M E N}

Se caracterizó la violencia intrafamiliar por tipo de familias, zona geográfica, tiempo de tratamiento y rangos de edad en una población pediátrica en consulta en el Servicio de la Cátedra de Psiquiatría. Estudio descriptivo, transversal, con muestreo consecutivo de 120 pacientes que solicitaron atención psicológica del 2010 al 2012. Se analizaron fichas clínicas, para el análisis el paquete estadístico SPSS versión 15.0 para Windows. Víctimas de violencia, (VV) $59.3 \%$ (86) y no víctimas (NV) $23.4 \%(34)$, Rango de edad de mayor violencia entre 15 a 18 años (34\% VV y $20 \%$ NV). Los de Asunción (59 \% VV y $21 \%$ NV) y ciudades cercanas ( $76 \%$ VV y $24 \% \mathrm{NV}$ ) mostraron tres veces más violencia que los del interior (61\% VV y $39 \% \mathrm{NV}$ ). Familias nucleares (36\% VV y $44 \% \mathrm{SV}$ ) niveles menores de violencia que monoparentales (38\% VV y $32 \% \mathrm{SV}$ ). Convivencia con otros parientes ( $14 \%$ VV y $21 \%$ SV) menor violencia; convivencia con extraños (6 \% VV y $3 \% \mathrm{SV}$ ) la duplica, así como población con padres con trastornos mentales ( $28 \%$ VV y $15 \%$ SV). Mayor tardanza para consultar, $41 \%$ (VV, $86 \%$ incluye abuso sexual). Mayor duración de tratamiento en $57 \%$ VV. Concentración de violencia en etapa adolescente, en sectores urbanos, en convivencia con extraños, familias monoparentales, familias con algún padre o madre con trastornos mentales. Tardanza a consultas en violencia por abuso sexual. La tendencia a vivir en familias nucleares, con otros familiares disminuiría la violencia.

Palabras clave: consulta ambulatoria, violencia intrafamiliar, niñez y adolescencia.

\section{Domestic violence according to socio demographic characteristics in a pediatric population}

\section{A B S T R A C T}

The domestic violence was characterizad by type of family, geographic area, treatment time and age ranges in a pediatric population that attended the Service of Psychiatry. It is a descriptive, cross-sectional study with a consecutive sampling of 120 patients from 2010 to 2012. Medical records were used and data were analyzed with SPSS for Windows. The $59,3 \%$ (86) was victim of violence (VV) and $23,4 \%$ (34) no victims of violence (NV). Age range with more violence was $15-18$ years (34\% VV and $20 \% \mathrm{NV}$ ). Those from Asunción (59\% VV and $21 \% \mathrm{NV}$ ) and close cities (76\% VV and 24\% NV) showed three more violence than those from the countryside (61\% VV and $39 \% \mathrm{NV}$ ). Nuclear families (36\% and $44 \%$ VV SV) lower levels of violence than monoparental families (38\% and $32 \% \mathrm{VV}$ SV). Living with other relatives ( $14 \%$ and $21 \%$ VV SV) less violence; living with strangers ( $6 \%$ and $3 \% \mathrm{VV} \mathrm{SV}$ ) doubled the violence as well as people with parents suffering mental disorders (28\% and 15\% VV SV). Delay to consult, $41 \%$ (VV, $86 \%$ included sexual abuse). Longer treatment in $57 \% \mathrm{VV}$. There was concentration of violence in adolescent stage, in urban areas, in coexistence with strangers, single parent families, families with a parent 
suffering mental disorders. There was a delay in consultations of sexual abuse. The trend to live in nuclear families, with relatives, would decrease violence.

\section{Keywords: outpatient clinic, domestic violence, childhood and adolescence INTRODUCCIÓN}

En 2006, Naciones Unidas publicó un informe internacional sobre los niños y la violencia doméstica señalando que 80.000 niños y niñas mueren anualmente en América Latina a consecuencia de la misma $(1,2)$. La violencia produce daño a su salud física y mental, desequilibra los ambientes donde desarrolla su aprendizaje y produce pérdidas en lo referente a la igualdad entre géneros.

Una de las formas más habituales es la violencia intrafamiliar(2). La violencia intrafamiliar ocurre cuando hay maltrato (por acción u omisión) entre los miembros de una familia, pudiendo ser de tipo físico, psicológico, por negligencia o abandono, sexual o económico $(2,3)$.

Sin importar a qué clase social pertenezcan, edad de sus integrantes o religión que profesen, la violencia intrafamiliar afecta a muchas familias en nuestros países y el mundo entero, ya que trasciende culturas (4).

La familia, definida ampliamente, tiene el mayor potencial para proteger a los niños contra todas las formas de la violencia, así también puede capacitar a los niños para protegerse. Un supuesto básico de la Convención sobre los Derechos del Niño (CCR) es que la familia es el entorno natural para el crecimiento y el bienestar de todos sus miembros - en particular para los niños - (5). Juega un rol fundamental en la formación de niños, niña y adolescente y cuando se transforma en un lugar donde ocurre violencia y malos tratos, es importante detectar a tiempo para proteger e impedir que se desarrollen repercusiones a la salud mental y la integridad física. Uno de los dilemas al que los padres, madres o cuidadores se enfrentan es el dilema que implica cuáles son las pautas adecuadas de crianza, por lo que se tiende a confundir la educación con las conductas violentas (2), y es vital para el buen desarrollo de los niños y niñas aprender a educar y no castigar con violencia.

Un estudio sobre actitudes que justifican la violencia domestica como predictores de castigos corporales demostró que las madres que creían que los maridos estaban justificados para golpear a sus esposas eran más propensas a creer que el castigo corporal es necesario para criar hijos y las madres que creían que los maridos estaban justificadas en golpear a sus esposas y que el castigo corporal es necesario para criar hijos eran más propensas a reportar que su hijo había sufrido agresión psicológica y violencia física. $(5,6)$.

Los parámetros de crianza y modelos de educación varían según las culturas, por lo que se hace una tarea difícil determinar donde terminan las buenas prácticas de crianza y donde comienza el maltrato.

Cuando un niño o niña es víctima de violencia, toda su vida se sitúa alrededor de esa situación, y se hace muy difícil el desarrollo normal, produciendo repercusiones físicas, psicológicas, sexuales, y reproductivas a corto y largo plazo que conllevan un elevado costo económico y social (4). Con respecto a Paraguay, Unicef 2012, (4), refiere que la pobreza afecta a un $45 \%$ de los niños paraguayos y el $61 \%$ de estos, es víctima de algún tipo de violencia intrafamiliar. El $35 \%$ de esos casos constituyen violencia física grave (7).

La consulta ambulatoria constituye un momento oportuno para prestar ayuda y resolver situaciones de violencia familiar. Como expresó el Dr. Anders Nordström en su función de Director General Interino de la Organización Mundial de la Salud (OMS) (5) "no importa si ocurre en la familia, en la escuela, en la comunidad, en una institución o en el ámbito laboral, los trabajadores de la salud se encuentran en primera línea de la respuesta a la violencia contra la infancia".

El propósito del presente estudio fue analizar las características de familias con y sin violencia intrafamiliar en el Servicio Ambulatorio de la Cátedra de Psiquiatría de la UNA en Paraguay.

\section{MATERIALES Y MÉTODOS}

El estudio fue descriptivo, trasversal, en una población pediátrica que recibió atención psicológica en el Servicio Ambulatorio de la Cátedra de Psiquiatría. Se analizaron los datos de la conformación de las características sociodemográficas del contexto familiar, tipos de 
familia, consignados en todas las fichas de pacientes entre 3 y 18 años que consultaron entre enero del 2010 y diciembre del 2012.

Esta revisión retrospectiva de las 145 fichas de pacientes permitió seleccionar 120 pacientes que contaban con información sobre exposición a violencia intrafamiliar, procediéndose a indagar sobre las características sociodemográficas, tiempo de inicio del problema por el cual habían acudido a consulta, tiempo que duró el tratamiento, presencia de un familiar con trastornos mentales y tiempo de tardanza para acudir a la primera consulta luego de iniciado el problema.

Se procedió posteriormente a analizar los distintos tipos de estructura familiar, utilizando la clasificación del estudio de UNICEF (3) clasificándolas como "nucleares, mononucleares, extendidas y con otros parientes". A lo cual se agregó una variable encontrada en el presente grupo de convivencia con "extraños". Además se utilizaron conceptos de violencia intrafamiliar de la clasificación propuesta por el grupo de trabajo sobre maltrato infantil dependiente del Ministerio de Salud de Chile (6) en: violencia o maltrato físico "toda lesión física no accidental producto de castigo único o repetido de magnitud y características variables"; de tipo emocional entendido como "hostigamiento verbal, habitual de un niño a través de insultos, criticas, descréditos y ridiculización, así como la indiferencia o rechazo implícito o explícito"; abandono o negligencia, considerado como "falta de protección y cuidados físicos al niño, de parte de sus figuras de custodia, así como el abandono de sus necesidades evolutivas por falta de estimulación cognitivas que no se deben a carencias extremas de recursos socioeconómicos"; y por último el abuso sexual "entre un niño y un adulto, aguda o crónica, incluida la explotación sexual". (6).

Los datos fueron cargados en una hoja de cálculo Excel y posteriormente analizados con el paquete estadístico SPSS versión 15.0 para Windows 7. Los resultados se expresaron en forma de frecuencia absoluta y relativa. Se respetó la confidencialidad de la información asignando códigos a las fichas de los pacientes.

\section{RESULTADOS}

Se realizó la revisión retrospectiva de las historias clínicas de 120 pacientes de los cuales, el $71,7 \%(n=86)$ reportó haber sido víctima de violencia y $28,3 \% \quad(n=34)$ no haberlo sido.

En la comparación de las características demográficas entre las víctimas y no de violencia se constató que la frecuencia de violencia fue mayor en el grupo entre 15 a 18 años con el $80,6 \%$. Con respecto a la procedencia, los residentes de Asunción mostraron la mayor proporción. Las familias nucleares presentaron niveles menores de violencia $(67,4 \%)$ que las monoparentales, también con los tenían convivencia con otros parientes o con extraños (Tabla 1).

Tabla 1. Características demográficas de las víctimas y no de violencia en la población pediátrica. $\mathrm{n}=120$

\begin{tabular}{llll}
\hline Características demográficas & $\mathbf{n}$ & $\begin{array}{l}\text { Víctima de } \\
\text { violencia } \\
\mathbf{n = 8 6}\end{array}$ & $\begin{array}{l}\text { No fue víctima de } \\
\text { Violencia } \\
\mathbf{n = 3 4}\end{array}$ \\
\hline $\begin{array}{llll}\text { Grupo etario } \\
\text { 3 a 5 }\end{array}$ & 15 & $11(73,3)$ & $4(26,7)$ \\
6 a 7 & 16 & $11(68,8)$ & $5(31,2)$ \\
9 a 11 & 23 & $16(69,6)$ & $7(30,4)$ \\
12 a 14 & 30 & $19(63,3)$ & $11(36,7)$ \\
$\quad$ 15 a 18 & 36 & $29(80,6)$ & $7(19,4)$ \\
Procedencia & & & \\
$\quad$ Asunción & 55 & $48(87,3)$ & $17(12,7)$ \\
$\quad$ Central & 36 & $26(72,2)$ & $10(27,8)$ \\
$\quad$ Interior & 18 & $11(61,1)$ & $7(38,9)$ \\
$\quad$ Exterior & 1 & $1(100)$ & \\
Tipo de Familia* & & & \\
$\quad$ Nuclear & 46 & $31(67,4)$ & $15(32,6)$ \\
$\quad$ Mono parenteral & 44 & $33(75,0)$ & $11(25,0)$ \\
$\quad$ Extendida/otros parientes/extraños & 25 & $18(72,0)$ & $8(32)$ \\
\hline * Sin datos= 2 & & &
\end{tabular}

* Sin datos $=2$ 
Con respecto al tiempo de inicio del problema y la primera consulta, se observó que aquellos que tardaron más de 2 años en acudir a la consulta desde el inicio del problema mostraron mayor frecuencia de violencia, 83,3\% (35). El tiempo que duró el tratamiento fue en mayor proporción entre 1 a 6 meses en quienes el 77,8\% (49) fue víctima de violencia. En relación al tratamiento, 19 pacientes ya lo tuvieron previamente y de estos, $78,9 \%$ (15) reportaron ser víctimas de violencia. Un 82,8\% (24) del total (29) que tenía un familiar con trastornos mentales fue víctima de violencia y $74,4 \%$ (61) del total (82) con problemas en el colegio, reportó ser víctima (Tabla 2).

Tabla 2. Factores asociados a la violencia. $n=120$

\begin{tabular}{|c|c|c|c|}
\hline & $\mathbf{n}$ & Víctimas de Violencia & $\begin{array}{l}\text { No fue víctima de } \\
\text { Violencia }\end{array}$ \\
\hline \multicolumn{4}{|c|}{$\begin{array}{l}\text { Tiempo transcurrido entre inicio } \\
\text { del problema y primera consulta }\end{array}$} \\
\hline$<1$ mes & 19 & $13(68,4)$ & $6(31,6)$ \\
\hline 1 a 6 meses & 30 & $19(63,3)$ & $11(36,7)$ \\
\hline 7 a 12 meses & 14 & $9(64,3)$ & $5(35,7)$ \\
\hline 13 a 24 meses & 8 & $4(50,0)$ & $4(50,0)$ \\
\hline$>24$ meses & 42 & $35(83,3)$ & $7(16,7)$ \\
\hline Sin especificar & 7 & $6(85,7)$ & $1(14,3)$ \\
\hline \multicolumn{4}{|c|}{ Tiempo que duró el tratamiento } \\
\hline 1 a 3 semanas & 44 & $28(63,6)$ & $16(36,4)$ \\
\hline 1 a 6 meses & 63 & $49(77,8)$ & $14(22,2)$ \\
\hline 7 a 12 meses & 8 & $4(50,0)$ & $4(50,0)$ \\
\hline$>12$ meses & 4 & $4(100,0)$ & \\
\hline Sin especificar & 1 & $1(100,0)$ & \\
\hline \multicolumn{4}{|c|}{ Con tratamiento previo } \\
\hline $\mathrm{Si}$ & 19 & $15(78,9)$ & $4(21,1)$ \\
\hline No & 92 & $63(68,5)$ & $29(31,5)$ \\
\hline Sin Datos & 9 & $8(88,9)$ & $1(11,1)$ \\
\hline \multicolumn{4}{|c|}{ Familiar con trastorno mental } \\
\hline $\mathrm{Si}$ & 29 & $24(82,8)$ & $5(17,2)$ \\
\hline No & 50 & $28(56,0)$ & $22(44,0)$ \\
\hline \multicolumn{4}{|c|}{ Problemas en el colegio } \\
\hline $\mathrm{Si}$ & 82 & $61(74,4)$ & $21(25,6)$ \\
\hline No & 28 & $16(57,1)$ & $12(42,9)$ \\
\hline No escolarizados & 10 & $9(90,0)$ & $1(10,0)$ \\
\hline
\end{tabular}

Entre quienes tuvieron la mayor tardanza para acudir a consulta luego de iniciado el problema se encontraron aquellos que tuvieron exposición al abuso sexual infantil, 85,7\% (12) del total (14) (Tabla 3).

Tabla 3. Tardanza de 2 años para acudir a consulta desde el inicio del problema.

\begin{tabular}{llll}
\hline & $\mathbf{n}$ & A dos años & Antes de 2 años \\
\hline Violencia psicológica & 59 & $22(37,3)$ & $37(62,7)$ \\
Violencia Física & 54 & $13(24,1)$ & $41(75,9)$ \\
Abuso sexual & 14 & $12(85,7)$ & $2(14,3)$ \\
Negligencia y Abandono & 6 & $2(33,3)$ & $4(66,7)$ \\
\hline
\end{tabular}




\section{DISCUSIÓN}

Más de la mitad de la población infanto-juvenil que acudió a consulta había sido víctima de violencia intrafamiliar, cifra que se encuentra dentro del rango de un estudio de UNICEF (3) y de otros estudios como uno de Alto Paraná, donde, de 1049 mujeres embarazadas, $41,8 \%$ había manifestado haber utilizado golpes con objetos como cintos y otros para disciplinar a sus hijos, $20,4 \%$ cachetadas, $51,2 \%$ gritos, $6,7 \%$ golpes de puño, entre otros (8) y al igual que un estudio probabilístico con 661 adolescentes de Alto Paraná donde, de un total de 661 adolescentes, 39,3\% recibió golpes con objetos, 16,4\% golpes de puño, $42,3 \%$ cachetadas, $55,5 \%$ gritos y $8,6 \%$ reportó abuso sexual, entre otros tipos de violencia de parte de familiares o encargados de su crianza (9).

La franja de mayor duración del tratamiento en la población se ubicó entre quienes padecieron violencia, lo que podría deberse a que las poblaciones víctimas de violencia presentan aumento del riesgo de padecer trastornos mentales como lo indican varios estudios (11-16).

En las familias donde se constató la presencia de violencia, las mismas caracterizaron por concentrarse en sectores urbanos más que los no urbanos. Este dato contrasta con los datos obtenidos en la encuesta de las Naciones Unidas (16), pero puede deberse a la mayor accesibilidad al servicio ambulatorio de la población de sectores urbanos.

Padres, madres y encargados de la crianza ejercieron violencia en mayor medida hacia adolescentes que hacia niños y niñas, lo que podría explicarse por la característica propia del proceso adolescente normal en el que los adolescentes requieren del ejercicio de la rebeldía y mayor libertad, manifestaciones propias de la reivindicación adolescente; que a su vez, llevadas a un contexto social con larga historia de autoritarismo y poca tolerancia a expresiones de libertad, provocaría la utilización de más violencia hacia ese rango de edad.

El estudio mostró además que la población infanto-juvenil, conviviendo en familias nucleares, está ligeramente menos expuesta que cuando conviven con uno de sus padres (familias monoparentales) y la violencia y aumentó cuando alguno de sus padres padecía algún trastorno mental. En estudios de Kaplan et al. de 1998 con jóvenes abusados físicamente, se comprobó que la exposición a ese tipo de violencia les dejaba una probabilidad siete veces mayor de desarrollar trastorno depresivo mayor que aquellos que no fueron maltratados y se advertía sobre el mayor riesgo al contar con un solo padre biológico en el hogar o vivir en familia tipo monoparental (10).

La mayor tardanza (más de dos años) para consultar ocurrió en los casos de abuso sexual. En el informe de las Naciones Unidas se estima un registro limitado de los casos de abuso debido al miedo de realizar las denuncias y la complejidad de los procesos que implican por lo cual los datos obtenidos en este estudio pueden entenderse por las mismas creencias e incluso se podría estimar que también puede existir un sub registro $(1,5$.

La convivencia con otros parientes mostró menor violencia comparada con la convivencia con extraños que la duplicó lo que coincide con el estudio de Unicef-Beca del 2010 (3).

No se encontró porcentaje diferencial significativo sobre niños, niñas y adolescentes víctimas de violencia con tratamiento previo. Al analizar y comparar los datos obtenidos hemos podido observar que la violencia continúa siendo un gran problema que debe ser abordado de una forma integral, con esfuerzos conjuntos no solo de los trabajadores de la salud mental si no desde todas las especialidades que trabajan con población vulnerable, ya que como hemos visto, las cifras son elevadas en general en Paraguay y se debe realizar una búsqueda de factores de riesgo y signos de violencia en cada niño o niña durante las consultas. La tarea consiste en sumar esfuerzos para evitar que la violencia ocurra, en primer lugar, y cuando esto no fuese posible, que la población infanto-juvenil afectada reciba los mejores servicios posibles para reducir los efectos perjudiciales (5).

Debe quedar claro para trabajadores y trabajadoras de la salud la importancia de frenar la violencia que los padres y madres o encargados de la crianza ejercen contra la población infantil. Aun cuando en Paraguay existe una justificación del maltrato o naturalización de la violencia hacia niños, niña y adolescente en Paraguay $(3,17,18)$, la violencia nunca puede ser justificada por razones disciplinarias o tradiciones culturales, tampoco se pueden aceptar niveles "razonables" de violencia por el peligro de una violencia legalizada que llevará indefectiblemente a la tolerancia de la violencia contra la infancia en general $(5,6,19)$. 


\section{REFERENCIAS BIBLIOGRAFICAS}

1. Secretaría Nacional de la Niñez y la Adolescencia [Internet]. Asunción-Paraguay: Movimiento Mundial por la Infancia y Secretaría Nacional de la Niñez y la Adolescencia; [citado 4 junio 2013]. Disponible en PDF: http://www.activamente.com.py/sites/default /files/hoja_datos_sobre_violencia_en_paragu ay.pdf

2. Larrain HS, Bascunan DC. Maltrato infantil y relaciones familiares en Chile: Análisis comparativo. Rev. chil. pediatr. 2008; 79(1):64-79.

3. UNICEF [Internet]. Asunción-Paraguay: Base Educativa y Comunitaria de Apoyo (BECA), UNICEF; [citado 11 de junio 2013]. Disponible en PDF: http://www.unicef.org/paraguay/spanish/py_ resources_Estudio_Maltrato.pdf

4. UNICEF [Internet]. Buenos Aires-Argentina: UNICEF, Ministerio de Desarrollo Social de la Provincia de Buenos Aires; [citado 10 junio 2013]. Disponible en PDF: http://www.unicef.org/argentina/spanish/abo rdajes.pdf

5. Fondo de las Naciones Unidas para la Infancia [Internet]. Ginebra-Suiza: Pinheiro PS; [citado 11 de junio 2013]. Disponible en PDF en:

http://www.unicef.org/lac/Informe_Mundial_ Sobre_Violencia_1\%281\%29.pdf

6. OMS, Oficina Regional para las Américas-OPS [Internet]. Washington, DC-EEUU: García Moreno C, Guedes A, Knerr W; [citado 11 de Junio 2015]. Disponible en PDF en: http://apps.who.int/iris/bitstream/10665/988 16/1/WHO_RHR_12.36_spa.pdf?ua=1

7. PARAGUAY.com [Internet]. AsunciónParaguay : UNICEF; [citado 12 de agosto de 2013]. Disponible en: http://www.paraguay.com/nacionales/pobrez a-afecta-al-45-de-los-ninos-paraguayos-y-laviolencia-al-61-82053

8. Arrom $C$, Ruoti $M$, Orué $E$, Arrom CM. El castigo y sus diferentes formas en la crianza de niños, niñas y adolescentes en 1049 familias de Alto Paraná. Revista Paraguaya de Psiquiatría 2015;3(1):26-32.

9. Ruoti M, Arrom C. Diferentes formas de violencia padecidas por adolescentes durante su crianza. Revista Paraguaya de Psiquiatría 2015; Volumen 3 (1):50-6.

10. Kaplan S, Pelcovitz D, Salzinger S, Weiner M, Mandel F, Lesser $M$ y col. Adolescent physical abuse: Risk for adolescent psychiatric disorders. The American Journal of Psychiatry 1998; 155 (7):954-60.
11. Organización Mundial de la Salud. Violencia contra la mujer. [Internet]. Ginebra-Suiza: OMS; [citado 15 mayo 2013]. Disponible en: http://www.who.int/mediacentre/factsheets/f s239/es/

12. Fresco MP, Arrom C, Samudio M, Arrom CM, Capurro M, Arrom MA, Arce A, Fresco M. Psicopatología en adultos con antecedentes de violencia intrafamiliar. Revista Paraguaya de Psiquiatría. 2015; 3(1):8-12.

13. Arrom C, Ruoti M, Samudio M, Orué $E$. Sintomatología depresiva y su relación con la edad y el sexo en adolescentes estudiantes de colegios secundarios de Alto Paraná. Revista Paraguaya de Psiquiatría. 2015; 3(1):33-7.

14. Arrom C, Fresco MP, Arrom CM, Arce A, Arrom MA, Romero M, Samudio M, Vargas V, Capurro M. Violencia intrafamiliar. Revista Paraguaya de Psiquiatría. 2015; 3(1):38-41.

15. UNICEF. Como eliminar la violencia en niños y niñas. [Internet]. Ginebra-Suiza: Unión Interparlamentaria (UIP), Oficina del Observador Permanente de la UIP, UNICEF; [citado 10 junio 2013]. Disponible en PDF: http://www.ipu.org/PDF/publications/violence es.pdf.

16. UNICEF. Estudio de violencia contra niños, niñas y adolescentes. [Internet]. República Dominicana: Paulo Pinheiro, Asamblea General de las Naciones Unidas 2006; [citado 10 junio 2014]. Disponible en PDF: http://www.unicef.org/republicadominicana/p rotection_8261.html.

17. Orue I, Calvete E. Justification of violence as a mediator between exposure to violence and agressive behavior in children. Psicothema [serial on the Internet]. (2012, Feb), [cited November 1, 2015]; 24(1):42-7. Available from: PsycINFO.

18. Valdebenito L, Larrain. S. El maltrato deja huellas. [Internet]. Santiago de Chile: Fondo de las Naciones Unidas para la Infancia (UNICEF), Programa Puente, Sistema de Protección Chile Solidario, Fondo de Solidaridad e Inversión Social, (FOSIS); [citado 12 junio 2013]. Disponible en:http://www.unicef.cl/archivos_documento /208/UNICEF\%20completo.pdf

19. González Vera, M. Patriarcado, machismo e impunidad: causas de la cotidiana violencia de género En: Coordinadora de Derechos Humanos del Paraguay (Codehupy). Derechos humanos en Paraguay/ Yvypóra Derécho Paraguáipe. Codehupy. Asunción: Mercurio S.A.; 2012. pp. 447-63. 